\title{
REPORTE DE CUATRO CASOS CLÍNICOS DE FILARIASIS EN ALTO NANAY, LORETO
}

\author{
Javier Vargas-Herrera ${ }^{1,2, a}$, Nancy Arróspide-Velasco ${ }^{1, b}$, Sonia Gutierrez-González, \\ Juan C. Celis-Salinas ${ }^{3, \mathrm{c}}$, Daniel Huamaní-Solano, ${ }^{4, \mathrm{~d}}$, Luis Loza-Hermenegildo ${ }^{3, \mathrm{e}}$, Julio Elgegren-Lao ${ }^{3,5, \mathrm{f}}$, \\ José Armas-Montes ${ }^{3,5,9}$, Juan Baca-Pérez ${ }^{3, \mathrm{~h}}$, César Cabezas ${ }^{1,2, \mathrm{c}}$
}

\section{RESUMEN}

El presente estudio describe los hallazgos clínicos, parasitológicos y de laboratorio de cuatro pacientes residentes en el distrito de Alto Nanay, Maynas, Loreto, Perú; infectados con microfilarias de Mansonella ozzardi. La evaluación clínica incluyó las especialidades de oftalmología, cardiología y nefrología. En el caso 1, destaca la presencia de dos tumoraciones subcutáneas, una a nivel dorsal y la otra en el tercio inferior de la pierna izquierda; en el caso 2, la sensación de "hormigueo o frío en las piernas"; en el caso 3 se encontró asociada una infección crónica por hepatitis B y en el caso 4 una tumoración de gran tamaño en región lumbar izquierda. En los cuatro pacientes se encontró Mansonella ozzardi y eosinofilia, tres presentaron valores mayores a $20 \%$. La tumoración en el caso 4 correspondió a una hernia de contenido intestinal. Es necesario ejecutar más estudios clínicos y evaluar su verdadero efecto patógeno, es también pertinente estudiar la diversidad genética de filarias de la Amazonia peruana.

Palabras clave: Filariasis; Patología clínica; Enfermedades desatendidas (fuente: DeCS BIREME).

\section{REPORT OF FOUR CLINICAL CASES OF FILARIASIS IN ALTO NANAY, LORETO}

\section{ABSTRACT}

This study describes the clinical, parasitological and laboratory findings of four patients who lived in the district of Alto Nanay, Maynas, Loreto, Peru and were infected with Mansonella ozzardi microfilariae. Clinical examinations by ophthalmologists, cardiologists and nephrologists were performed. In case 1, the presence of 2 subcutaneous lumps was the most important finding, one at dorsal level and the other in the lower third of the left leg; in case 2, there was a sensation of tingling or coldness in the legs; in case 3, an associated chronic hepatitis B infection was found, and in case 4, a large lump was detected in the left lumbar region. All 4 patients were infected with Mansonella ozzardi and had eosinophilia, 3 of them in percentages of $20 \%$. The lump found in case 4 was due to a herniation of bowel content. Further clinical studies and an evaluation of the actual pathogenic effect of microfilariae are to be performed. A study of the genetic diversity of filariae in the Peruvian Amazon would also be important.

Key words: Filariasis; Pathology, clinical; Neglected diseases (source: MeSH NLM).

\section{INTRODUCCIÓN}

La filariasis es una parasitosis producida por nemátodos hemáticos de la familia Filariidae. Las filarias son nemátodos tisulares transmitidos por picaduras de mosquitos hematófagos (1). De acuerdo con la especie de filaria y la región anatómica que afectan se clasifican en: linfáticas, subcutáneas y de cavidades serosas ${ }^{(2)}$.
Las filariasis de cavidades serosas son causadas por Mansonella perstans y Mansonella ozzardi; son citadas en los textos de parasitología como asintomáticas o relacionadas con síntomas inespecíficos como dolor osteomuscular, fatiga, dermatitis, etc. sin embargo, existen reportes que las relacionan con hidrocele o engrosamiento de ganglios linfáticos ${ }^{(1-4)}$. La filaria de más amplia distribución en América es Mansonella ozzardi, se han reportado casos en México, países

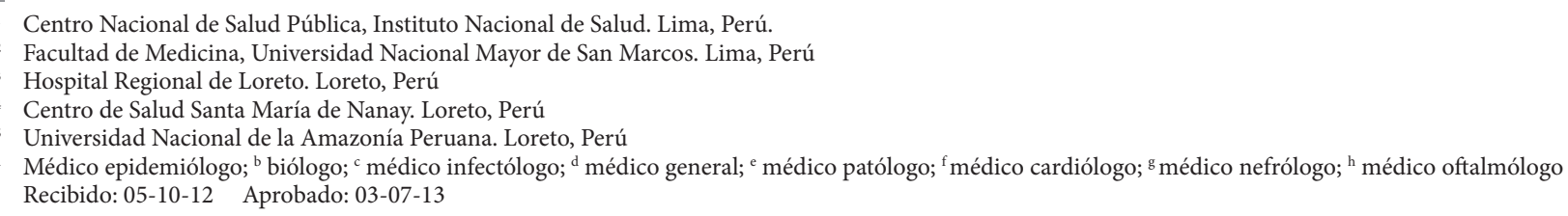

Citar como: Vargas-Herrera J, Arróspide-Velasco N, Gutierrez-González S, Celis-Salinas JC, Huamaní-Solano D, Loza-Hermenegildo L, et al. Reporte de cuatro casos clínicos de filariasis en Alto Nanay, Loreto. Rev Peru Med Exp Salud Publica. 2013;30(3):506-11. 
de Centroamérica y del Caribe, Venezuela, Surinam, Guyana Francesa, Brasil, Argentina, Bolivia y Perú (1).

En el Perú, el primer reporte de Mansonella ozzardi documentado, data de 1958 cuando el doctor Gonzales-Mugaburu reportó su presencia en cuatro personas adultas residentes en el Asilo de Ancianos de Iquitos ${ }^{(5)}$. Desde entonces se han publicado como hallazgos incidentales en reportes de diferentes localidades del departamento de Loreto ${ }^{(6-8)}$. En años más recientes, 2009 y 2010 se han venido identificando casos de filariasis en localidades rurales cercanas a la ciudad de lquitos, refiriendo una procedencia de localidades del distrito de Alto Nanay ${ }^{(9)}$.

En noviembre de 2010, el Instituto Nacional de Salud realizó en Santa María de Nanay, distrito de Alto Nanay, una búsqueda activa de casos de malaria. Se examinaron a 164 pobladores mediante pruebas de gota gruesa y frotis. La mayoría de las láminas fueron negativas para malaria, sin embargo, se identificaron 21 casos de infección por microfilarias, con una prevalencia de 12,8\%.

Durante la segunda semana de octubre de 2011, cuatro pacientes varones de 36 a 69 años, residentes en el distrito de Santa María de Nanay, provincia de Maynas, departamento de Loreto fueron atendidos en el Hospital Regional de Loreto, en el distrito de Punchana, ciudad de Iquitos, referidos por el Centro de Salud de Santa María de Nanay. Los pacientes fueron evaluados sucesivamente en los consultorios de infectología, cardiología, nefrología y oftalmología. Adicionalmente se les solicitaron los siguientes exámenes auxiliares: gota gruesa y frotis de sangre, hemograma completo, bioquímica hemática, examen completo de orina, radiografía de tórax y pulmones, y ecografía abdominal. Adicionalmente, el caso cuatro fue sometido a una tomografía espiral multicorte por un tumor en la región lumbar. Los exámenes médicos especializados en cardiología incluyeron un electrocardiograma y un ecocardiograma; en oftalmología, un examen de agudeza visual, y anexos como la exploración del segmento anterior, refracción, tono ocular y fondo de ojo con el uso de un agente midriático tópico.

Los exámenes parasitoscópicos hemáticos fueron realizados en el Instituto Nacional de Salud, siguiendo el método de identificación de filarias descrito por Thomas C. Orihel and Mark L. Eberhard.1982 (10). Los otros exámenes se realizaron en el laboratorio del Hospital Regional de Loreto y en los casos en que no estuvo disponible el servicio, en un laboratorio particular.

Los pacientes firmaron un consentimiento informado para la extracción de sangre destinado a la investigación de malaria, como parte de los procedimientos para la elaboración de paneles de láminas de gota gruesa y frotis del Programa de Evaluación Externa del Desempeño en el diagnóstico de Malaria que realiza el Instituto Nacional de Salud y la Organización Panamericana de la Salud. Bajo estas circunstancias, se realizaron los hallazgos de filariasis por Mansonella ozzardi y su posterior referencia del Centro de Salud de Santa María de Nanay al Hospital Regional de Loreto.

Todos los pacientes fueron sometidos a procedimientos de diagnóstico y recibieron indicaciones para el tratamiento y seguimiento de las enfermedades diagnosticadas, de acuerdo con los protocolos de atención establecidos por el servicio de salud. El presente estudio se limitó a la descripción de los hallazgos encontrados.

\section{REPORTE DE CASOS}

Se estudiaron cuatro varones infectados con microfilarias de Mansonella ozzardi (Figura 1), todos residentes en el distrito de Alto Nanay en las localidades de Santa María de Nanay y en caseríos cercanos ubicados en los ríos Chambira o Pintuyacu. Las edades de los pacientes fueron: 36, 49, 68 y 69 años. Todos refirieron haber nacido en lquitos, pero el tiempo de residencia en el Alto Nanay es mayor de 30 años en todos los casos. Así mismo, todos refieren haber presentado al menos un episodio de malaria en los últimos 12 meses previos al examen clínico.

En la anamnesis acerca de la enfermedad actual se identificaron cefalea y dolores osteomusculares en los tres primeros casos, principalmente en la región lumbar. Prurito y erupción dérmica eritematosa en el primer caso; episodios de sensación de "hormigueo o frío en las piernas" en el segundo caso; y obnubilación, mareos en el tercer caso. El cuarto caso no manifestó molestias.

El examen físico en el primer caso, reveló la presencia de una tumoración subcutánea dorsal en la región paravertebral superior derecha, blanda, móvil, ovalada de $6 \mathrm{~cm}$ por $3 \mathrm{~cm}$; y otra tumoración en el tercio inferior, cara externa de la pierna izquierda, blanda, móvil, circular de $2 \mathrm{~cm}$ por $2 \mathrm{~cm}$. El examen físico del segundo caso reveló una tumoración de forma arriñonada, de gran tamaño: $17 \mathrm{~cm}$ de largo por $12 \mathrm{~cm}$ de ancho y de $9 \mathrm{~cm}$ de espesor, localizada en la región lumbar izquierda, de consistencia blanda y poco móvil (Figura 2). En ninguno de los casos se estableció, mediante exámenes anatomopatológicos, que las tumoraciones descritas se encuentren relacionadas con la infección por microfilarias. 

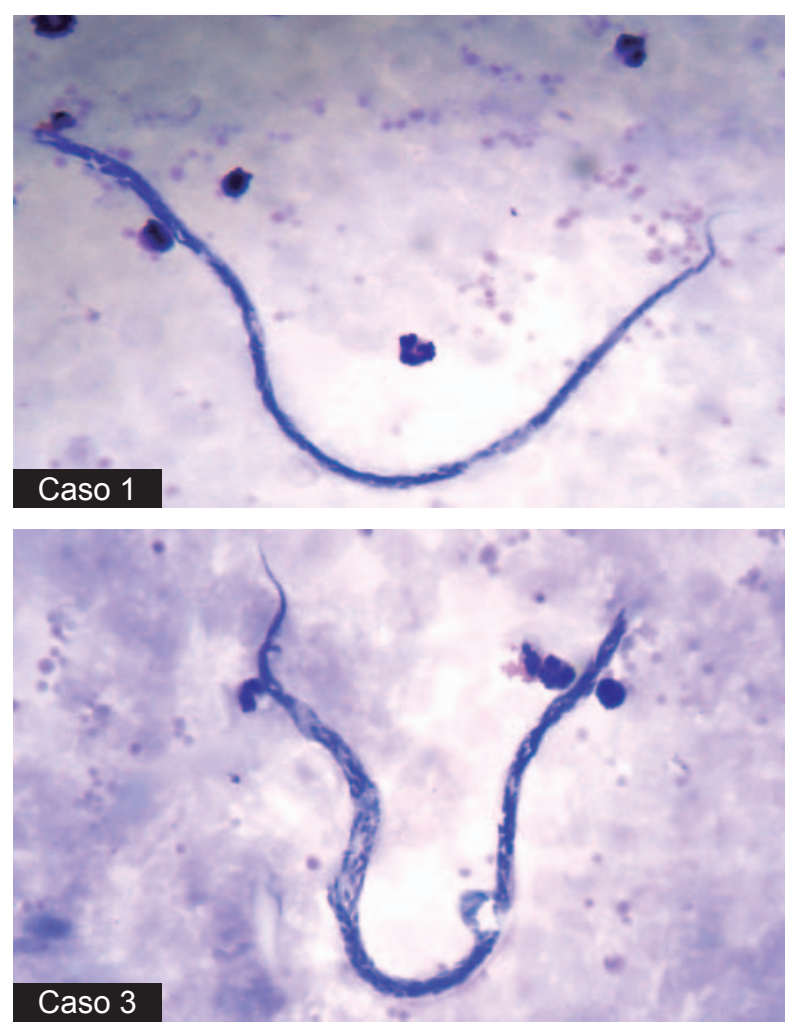
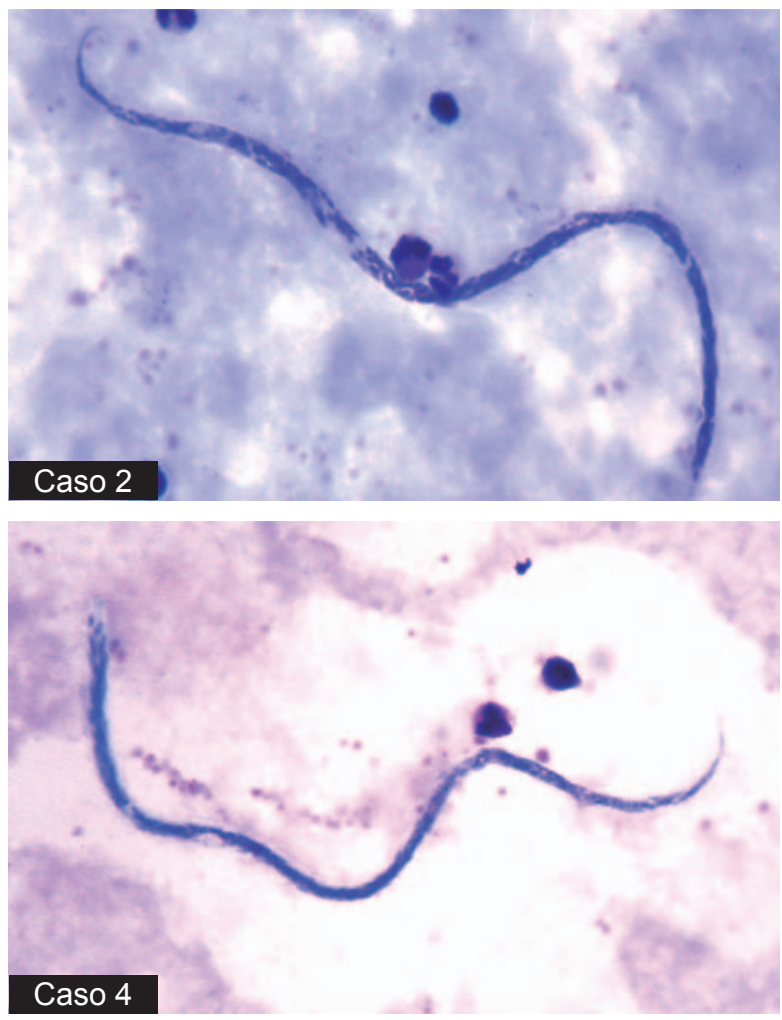

Figura 1. Microfilarias de Mansonella ozzardi encontrados en examen de gota gruesa (Giemsa, 100x)

Los resultados de los exámenes de laboratorio practicados muestran los valores de leucocitos dentro de parámetros normales para los cuatro casos. Tres de los cuatro casos presentaron valores de hemoglobina menor a $14 \mathrm{~g} / \mathrm{dL}$, sin embargo, ninguno puede ser considerado como caso de anemia.

Las densidades parasitarias para microfilarias variaron de 1 a 2 hasta más de 40 parásitos por campo. Todos los casos presentaron valores de eosinófilos por encima del parámetro normal, tres de ellos con valores que cuadruplican y quintuplican el valor máximo de dicho parámetro. El mayor grado de parásitos por

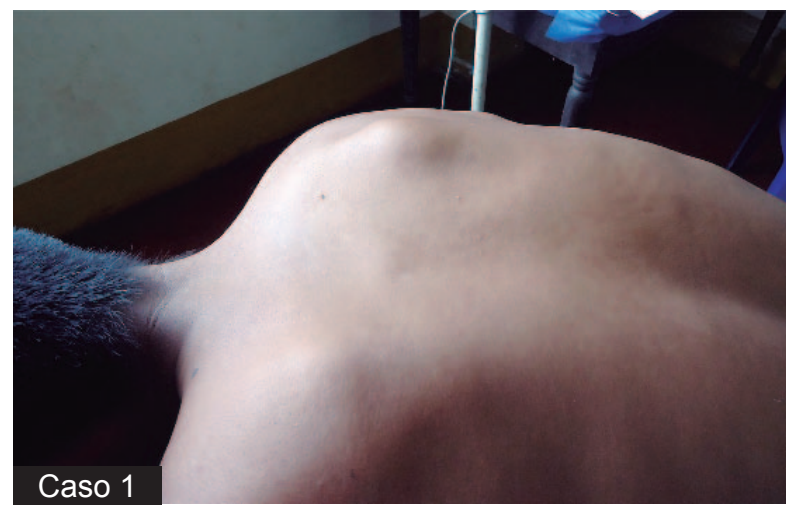

Figura 2. Paciente con tumoración nodular en región dorsal campo se relacionó con presentar mayor recuento de eosinófilos $(25,8 \%)$ y síntomas más específicos como la sensación de "hormigueo en las piernas". Los otros exámenes auxiliares tuvieron diferentes resultados para cada uno de los casos estudiados. El tercer caso presentó en la ecografía, hepatomegalia con dilatación de la vena cava y de las venas intrahepáticas, lo que se correlacionó con el hallazgo de antígeno de superficie para hepatitis viral B positivo. La gran tumoración que presentó el cuarto caso correspondió a una hernia lumbar adquirida, diagnosticada por tomografía espiral multicorte, la cual fue corregida quirúrgicamente, no se reportó en la intervención la presencia de filarias en cavidad abdominal. La evaluación oftalmológica descartó la presencia de oncocercosis. Los casos dos, tres y cuatro presentan hallazgos oftalmológicos compatibles con la exposición a agentes irritantes ambientales y al proceso de envejecimiento (conjuntivitis, pterigion o cataratas). En el examen cardiovascular, el caso dos presentó esclerosis aórtica, pero con función cardiovascular dentro de parámetros normales; los casos tres y cuatro presentaron insuficiencia mitral leve y estenosis aórtica leve, respectivamente. En el examen nefrológico el caso cuatro presentó una reacción positiva para la detección de microalbuminuria mediante una tira reactiva en una muestra de orina (Tabla 1). 
Tabla 1. Hallazgos principales en los cuatro casos de filariasis

\begin{tabular}{|c|c|c|c|c|}
\hline Hallazgos & Caso 1 & Caso 2 & Caso 3 & Caso 4 \\
\hline Edad en años & 36 & 49 & 68 & 69 \\
\hline $\begin{array}{l}\text { Filariasis } \\
\text { Densidad parasitaria } \\
\text { (por campo) }\end{array}$ & De 1 a 2 & Más de 40 & De 10 a 15 & De 1 a 2 \\
\hline Eosinófilos (\%) & $(20,6)$ & $(25,8)$ & $(20,8)$ & $(9,5)$ \\
\hline Ecografía abdominal & $\begin{array}{l}\text { Sin alteraciones } \\
\text { patológicas }\end{array}$ & $\begin{array}{l}\text { Sin alteraciones } \\
\text { patológicas }\end{array}$ & $\begin{array}{l}\text { Hepatomegalia, vena } \\
\text { cava dilatada, dilatación } \\
\text { de venas intrahepáticas }\end{array}$ & Litiasis vesicular \\
\hline $\begin{array}{l}\text { Tomografía de tumo- } \\
\text { ración }\end{array}$ & - & - & - & $\begin{array}{l}\text { Tumor en región lumbar izquierda. Presencia de } \\
\text { solución de continuidad en región lumbar, con salida } \\
\text { de contenido intestinal y asas intestinales en rela- } \\
\text { ción a hernia, la misma que se extiende por tejido } \\
\text { celular subcutáneo por la región posterior del tórax }\end{array}$ \\
\hline Ag de superficie HVB & Negativo & Negativo & Positivo & Negativo \\
\hline $\begin{array}{l}\text { Examen } \\
\text { oftalmológico }\end{array}$ & $\begin{array}{l}\text { Examen oftalmo- } \\
\text { lógico normal }\end{array}$ & $\begin{array}{l}\text { Pterigion en } \\
\text { ambos ojos }\end{array}$ & $\begin{array}{l}\text { Miopía, pterigion en } \\
\text { ambos ojos }\end{array}$ & $\begin{array}{l}\text { En ambos ojos: conjuntivitis inespecífica, obstruc- } \\
\text { ción de puntos lagrimales, degeneración macular } \\
\text { en relación con edad, catarata incipiente }\end{array}$ \\
\hline $\begin{array}{l}\text { Examen } \\
\text { cardiológico }\end{array}$ & $\begin{array}{l}\text { Función } \\
\text { cardiovascular } \\
\text { normal }\end{array}$ & $\begin{array}{c}\text { Esclerosis aór- } \\
\text { tica con función } \\
\text { cardiovascular } \\
\text { normal }\end{array}$ & $\begin{array}{l}\text { Bradicardia sinusal, insu- } \\
\text { ficiencia mitral leve }\end{array}$ & $\begin{array}{l}\text { Crecimiento de aurícula izquierda, } \\
\text { estenosis aórtica leve }\end{array}$ \\
\hline $\begin{array}{l}\text { Examen } \\
\text { nefrológico }\end{array}$ & $\begin{array}{c}\text { Función renal } \\
\text { normal }\end{array}$ & $\begin{array}{c}\text { Función renal } \\
\text { normal }\end{array}$ & Función renal normal & Signos de microalbuminuria \\
\hline
\end{tabular}

\section{DISCUSIÓN}

La prevalencia de la infección por filarias de la especie Mansonella ozzardi en diversas localidades de la región Loreto en la Amazonía peruana ha sido documentada desde $1958{ }^{(5)}$. Recientemente, Chuquicaña et al. (11) reportaron que las prevalencias en algunas localidades ribereñas cercanas a lquitos están alrededor de 1\%; que en localidades rurales ubicadas en los ríos Yavarí, la prevalencia alcanza el $35 \%$, y que en comunidades ubicadas en los ríos Putumayo y en el río Pintuyacu del distrito de Alto Nanay, las prevalencias son mayores de $60 \%$. La filariasis es endémica también en localidades de la Amazonía brasileña ${ }^{(12-14)}$ limítrofes con el Perú, con prevalencias que alcanzan hasta el $48 \%$ en algunas de ellas.

Las localidades que presentan elevadas prevalencias para filariasis, son áreas endémicas o hiperendémicas de malaria. El $70 \%$ de los casos de malaria en el país, proceden del departamento de Loreto ${ }^{(15)}$, por lo que no es extraño que el hallazgo de filarias sea incidental al investigar malaria o se encuentre en coinfección con plasmodios ${ }^{(9,16)}$. Los cuatro casos estudiados que motivan el presente reporte, refirieron antecedentes de malaria y son residentes del distrito de Alto Nanay, reconocida área hiperendémica de malaria con un índice palúdico anual mayor a 50 por cada 1000 habitantes ${ }^{(15)}$. Tres de ellos residen en la localidad de Santa María de Nanay, en la cual hemos observado una elevada prevalencia de filariasis y uno en la localidad de Saboya ubicada en el río Pintuyacu, en donde la prevalencia sería bastante mayor ${ }^{(11)}$.
A pesar de la elevada prevalencia de filariasis en la región Loreto, no se cuenta con reportes de signos y síntomas que permitan caracterizar un cuadro clínico en las personas infectadas con filarias, ni de las complicaciones médicas o secuelas que esta infección pudiera producirles. En general, se describe la infección como asintomática o con síntomas inespecíficos (1-3), sin embargo, ya en 1960, Batista et al. (17), documentaron una diferencia estadísticamente significativa de la presencia de dolores articulares, frialdad en las piernas, adenitis inguinocrurales, placas eritematosas y pruriginosas, y dolor de cabeza, entre los pacientes infectados y personas libres de la infección. El mismo autor refiere que el cuadro clínico es más evidente en los pacientes que muestran una mayor densidad parasitaria. Hallazgos similares se reportaron en 1999 en la región de El Chaco en Bolivia ${ }^{(18)}$ y más recientemente en Coari en la selva brasileña ${ }^{(13)}$.

En el presente estudio, los tres primeros casos presentaron cefalea y dolor osteomuscular en la región lumbar. Adicionalmente, el primer caso refirió episodios frecuentes de erupción dérmica acompañada de prurito, mientras que el segundo caso, sensación de "hormigueo o frialdad en las piernas". En contraste, el cuarto caso no refirió mayor sintomatología. Esta descripción es concordante con el cuadro clínico propuesto por Batista et al (13), cabe anotar que el cuadro clínico más evidente lo presentó el caso dos, quien además presentó la densidad parasitaria más alta. 
Entre los hallazgos del examen físico se destaca la presencia de tumores en dos pacientes. En el primer caso se trató de dos tumoraciones subcutáneas, una en la espalda y otra en la pierna, conocidos en el medio local como "lobanillos" que se ha observado también en otros pobladores infectados con filarias del distrito de Alto Nanay, y que describen también Chuquicaña y colaboradores ${ }^{(11)}$; sin embargo, en el presente estudio no se ha podido demostrar su relación con la infección por filarias. En el caso de infecciones por Mansonella perstans están descritas inflamaciones subcutáneas, pero no para la Mansonella ozzardi, por lo que es necesario establecer en subsiguientes estudios si este hallazgo es coincidente o está asociado con esta mansoneliasis. En el cuarto caso se trató de una hernia intestinal que migró progresivamente, tras varias décadas, de la región abdominal a la región lumbar como se muestra en la fotografía, y que corresponde a un hallazgo incidental.

De los resultados de los exámenes hematológicos y bioquímicos, destaca claramente el recuento de eosinófilos elevado encontrado en los cuatro casos y, particularmente, cuatro o cinco veces su valor relativo en los tres primeros casos. Este hallazgo está descrito en muchas enfermedades parasitarias (1-3) y también en las filariasis por Mansonella ozzardi, por lo que, la presencia de eosinofilia en residentes de Loreto debería sugerir la presencia de mansoneliasis en la discusión del diagnóstico diferencial de la eosinofilia elevada ${ }^{(19,20)}$.

La filariasis por Onchocerca está bastante reconocida como causa de ceguera, sin embargo, también se han documentado casos de queratitis corneal debido a la Mansonella ozzardi en poblaciones amazónicas brasileñas en quienes se descartó oncocercosis (20-23); Cohen et al. describieron molestias oculares en más de $70 \%$, y queratitis en más de $12 \%$ de los infectados por Mansonella ozzardi (21). Vianna et al demostraron una asociación entre la positividad a mansoneliasis y la positividad a lesiones corneales ${ }^{(22)}$. Las alteraciones oftalmológicas encontradas en los cuatro pacientes del presente estudio fueron las esperables en personas expuestas a irritantes ambientales o propios de los procesos degenerativos.

Por otra parte, la eosinofilia persistente que se encuentra en personas infectadas con Mansonella ozzardi puede ser un potencial patógeno de varios órganos, como corazón, pulmones o el aparato genitourinario. Probablemente el más grave sea el daño del endocardio y la subsecuente miocarditis ${ }^{(24)}$, así mismo se ha descrito una hipereosinofila subsecuente a una infección por Mansonella perstans en un niño de 11 años, a la que se le atribuye una insuficiencia mitral severa ${ }^{(25)}$. Las alteraciones de la función cardiaca encontradas en los casos tres y cuatro del presente estudio no pueden atribuirse a la infección por filarias y se explican en las lesiones encontradas, las cuales son frecuentes en personas mayores de 60 años.

En el caso tres se encontró antígeno de superficie positivo para hepatitis viral B y la ecografía abdominal reveló hepatomegalia y dilatación de la vena cava y de las venas intrahepáticas, lo cual confirma una infección crónica de hepatitis viral B. Estos hallazgos nos advierten que en los pobladores de la selva, especialmente de zona rural es frecuente que existan coinfecciones con otros agentes bastante prevalentes.

El estudio del perfil clínico de cuatro pacientes infectados con Mansonella ozzardi indica que los síntomas son inespecíficos, los cuales también pueden estar presentes en otras enfermedades tropicales prevalentes en la región, como dengue o malaria. En lo que respecta a la densidad parasitaria, se ha observado que su mayor cuantificación puede dar lugar a mayor sintomatología y a valores más elevados de eosinofilia.

La búsqueda de infección por filarias en láminas de gota gruesa debe tenerse en cuenta especialmente en personas sintomáticas residentes en áreas endémicas de malaria que son negativas a malaria. Finalmente, consideramos que otros temas pendientes en la agenda de investigación de esta parasitosis, que parece ser re emergente en la región Loreto, son: la distribución geográfica en la Amazonia peruana, la incriminación de un vector transmisor y el tratamiento racional, especialmente definiendo los criterios para ofrecer tratamiento.

Agradecimientos: al Dr. Pedro Valencia por brindar las facilidades logísticas para el traslado del equipo de investigación a las localidades en estudio. A Melitón Gebol Cahuaza y Franklin Chirinos Palomino por su apoyo en la búsqueda de casos, y diagnostico microscópico primario de los pacientes estudiados. A los pobladores de la comunidad de Santa María de Nanay.

Contribuciones de autoría: JVH participo en la concepción y diseño del artículo, la recolección de resultados, análisis e interpretación de datos y la redacción del artículo. NAV en la recolección e interpretación de datos y revisión crítica del artículo, SGG y DHS en la recolección e interpretación de datos y aporte de pacientes. JCCS, LLH, JEL, JAM y JBP en la recolección e interpretación de datos, CCS participó en la concepción del artículo y la aprobación de su versión final.

Fuentes de financiamiento: autofinanciado.

Conflictos de interés: los autores declaran no tener conflictos de interés en la publicación de este artículo. 


\section{REFERENCIAS BIBLIOGRÁFICAS}

1. Botero D, Marcos R. Filariosis, capítulo 11. En: Botero D, Marcos R. Parasitosis Humanas. 3ra ed. Medellín: Editorial Corporación para Investigaciones Biológicas; 1998.

2. Duménigo Ripoll B. Filariasis, capítulo 101. En: Llop Hernández A, ValdésDapena Vivanco M, Zuazo Silva JL. Microbiología y Parasitología Médicas. 1ra Ed. La Habana: Editorial Ciencias Médicas; 2001.

3. Pezzani BC. Filarias, capítulo 142. En: Basualdo JE, Coto CE, De Torres RA. Microbiología Biomédica. Buenos Aires: Editorial Atlante; 2006.

4. World Health Organization (WHO). Lymphatic Filariasis. Progress report 2000-2009 and strategic plan 20102020 of the global programme to eliminate lymphatic filariasis: halfway towards eliminating lymphatic filariasis. Geneva: WHO; 2010.

5. Gonzales-Mugaburu L. Hallazgo de Mansonellaozzardi en la Selva peruana. Nota preliminar. Rev Peru Med Exp Salud Publica. 1958;12(1-2):87-9.

6. Loja Oropeza D, Necochea Villafuerte Y, Vilca Vásquez M, Avilés Gonzaga R. Filariasis en el Perú: perfil clínicoepidemiológico. Folia Dermatológica Peruana. 1999;10(2 -3).

7. Zerpa R, Chuquicaña A. Microfilaria Mansonella ozzardi [galería fotográfica]. Rev Peru Med Exp Salud Publica. 2007;24(4):437-9.

8. Beltrán $\mathrm{M}$, Cancrini $\mathrm{G}$, Reátegui $\mathrm{G}$, Melgar R, Ayllón C, Garaycochea MC, et al. Filariosis humana en la selva peruana: reporte de tres casos. Rev Peru Med Exp Salud Publica. 2008;25(2):257-60.

9. Arróspide N, Adami YL, Durand S, Rimarachín D, Gutiérrez S, Cabezas C. Microfilaria atípica en coinfección con Mansonella ozzardi y Plasmodium vivax en la Amazonía peruana. Rev Peru Med Exp Salud Publica. 2009;26(3):412-3.
10. Orihel TC, Eberhard ML. Mansonella ozzardi: a redescription with comments on its taxonomic relationships. Am J Trop Med Hyg. 1982;31(6):1142-7.

11. Chuquicaña A, Durand S, Bentley G, Sanchez JF, Yalta A, Zerpa R, et al. Prevalencia y distribución de filariasis por Mansonella ozzardi en Cuenca Amazónica Peruana. I Conferencia Anual de la Sociedad Norteamericana de Medicina Tropical e Higiene en el Perú. Lima: Sociedad Norteamericana de Medicina Tropical e Higiene; Febrero de 2011.

12. Medeiros JF, Py-Daniel V, Barbosa UC, Izzo TJ. Mansonella ozzardi in Brazil: prevalence of infection in riverine communities in the Purus region, in the state of Amazonas. Mem Inst Oswaldo Cruz. 2009;104(1):74-80.

13. Martins M, Pessoa FA, de Medeiros MB, de Andrade EV, Medeiros JF. Mansonella ozzardi in Amazonas, Brazil: prevalence and distribution in the municipality of Coari, in the middle Solimões River. Mem Inst Oswaldo Cruz. 2010;105(3):246-53.

14. Medeiros JF, Py-Daniel V, Barbosa UC. Prevalence of Mansonella ozzardi among riverine communities in the municipality of Lábrea, State of Amazonas, Brazil. Rev Soc Bras Med Trop. 2011;44(2):186-90.

15. Perú, Ministerio de Salud, Dirección General de Epidemiología. Situación de la Malaria en el Perú. Boletín Epidemiológico. 2012;21(16):269-70.

16. Mollinedo S, De La Cruz L, Chavez T, Holguin E, Gironda W. Mansonella ozzardi: un potencial parásito emergente en Bolivia. Informe Técnico $\mathrm{N}^{\circ} 04 \mathrm{La}$ Paz: Instituto Nacional de Laboratorios de Salud; 2000.

17. Batista D, Oliveira W y Rabello V. Estudo da patogenicidade da Mansonellaozzardi e da sintomatologia da mansonelose. Rev Inst Med Trop Sao Paulo. 1960;2(5):281-9.
18. Bartoloni A, Cancrini G, Bartalesi F Marcolin D, Roselli M, Arce CC, et al. Manzonella ozzardi infection in Bolivia: prevalence and clinical associations in the Chaco region. Am J Trop Med Hyg. 1999;61(5):830-3.

19. McNeeley DF, Raccurt CP, Boncy J, Lowrie RC Jr. Clinical evaluation of Mansonella ozzardi in Haiti. Trop Med Parasitol. 1989;40(2):107-10.

20. Garrido C, Campos M. First report of presumed parasitic keratitis in Indians from the Brazilian Amazon. Cornea. 2000;19(6):817-9.

21. Cohen JM, Ribeiro JA, Martins M. Acometimento ocular em pacientes com mansonelose. Arq Bras Oftalmol. 2008;71(2):167-71.

22. Vianna LM, Martins M, Cohen MJ, Cohen JM, Belfort R Jr. Mansonella ozzardi corneal lesions in the Amazon: a cross-sectional study. BMJ Open. 2012;2(6). pii: e001266. doi: 10.1136/ bmjopen-2012-001266.

23. Branco BC, Chamon W, Belfort Neto R, Belfort JR, Costa JA. Achados oculares entre habitantes do município de Pauini e possível associação entre lesões corneanas e mansonelose na Amazônia. Arq Bras Oftalmol. 1998;61(6):67482.

24. Nutman TB. Evaluation and differential diagnosis of marked, persistent eosinophilia. Immunol Allergy Clin North Am. 2007;27(3):529-49.

25. Pavlovic M, Berdat P, Holzer B, Aebi C, Carrel T, Pfammatter JP. Severe mitral valve involvement in a child with hypereosinophilia secondary to parasitic infection. J Heart Valve Dis. 2003;12(5):649-51.

Correspondencia: Javier Vargas Herrera Dirección: Calle Cápac Yupanqui 1400, Lima 11, Perú.

Teléfono: (511) 748111 Anexo:2166

Correoelectrónico:juargas@ins.gob.pe 